\title{
Bases for time-resolved probing of transient carrier dynamics by optical pump-probe scanning tunneling microscopy
}

\author{
Munenori Yokota $^{a}$, Shoji Yoshida ${ }^{a}$, Yutaka Mera ${ }^{b}$, Osamu Takeuchi ${ }^{a}$, Haruhiro Oigawa ${ }^{a}$, and Hidemi \\ Shigekawa ${ }^{* a}$
}

\author{
5 Received (in $X X X, X X X)$ Xth $X X X X X X X X X 20 X X$, Accepted Xth $X X X X X X X X X 20 X X$ \\ DOI: $10.1039 / b 000000 x$
}

\begin{abstract}
The tangled mechanism that produces optical pump-probe scanning tunneling microscopy spectra from semiconductors was analyzed by comparing model simulation data with experimental data. The nonlinearities reflected in the spectra, namely, the excitations generated by paired laser pulses with a 10 delay time, the logarithmic relationship between carrier density and surface photovoltage (SPV), and the effect of the change in tunneling barrier height depending on SPV, were examined along with the delaytime-dependent integration process used in measurement. The optimum conditions required to realize reliable measurement, as well as the validity of the microscopy technique, were demonstrated for the first time.
\end{abstract}

\section{${ }_{15}$ Introduction}

With the current progress in manufacturing quantum functions in small organized structures, probing of quantum dynamics with high temporal and spatial resolutions has never been more crucial for further advances in nanoscale science and technology.

${ }_{20}$ Scanning tunneling microscopy (STM) has an excellent spatial resolution and has been widely used in atomic-scale research since its invention ${ }^{1,2}$. However, since its temporal resolution is generally limited to the millisecond range, the development of a new microscopy technique has been desired. Considerable effort

25 has been devoted to achieving this, and various methods and techniques have been developed ${ }^{3-18}$.

Recently, a microscopy technique that simultaneously realizes the spatial resolution of STM and the temporal resolution of ultrashort-pulse laser technology has been developed ${ }^{19-30}$. The 30 combination of STM with optical technology has advantages that enable the analysis of photoinduced dynamics on the nanoscale as well as the realization of ultrafast time-resolved microscopy.

In laser-combined STM, a nonequilibrium carrier distribution is generated using ultrashort laser pulses, and its relaxation 35 processes are probed by STM using the optical pump-probe (OPP) method realized in STM. For a semiconductor sample, two components, i.e., bulk-side decay and surface-side decay, which respectively reflect the decay of photocarriers on the bulk side and the decay of minority carriers tentatively trapped at the 40 surface, are observed ${ }^{23-26}$. By applying the microscopy technique, fundamental carrier dynamics in semiconductors were successfully probed and visualized, using the two-type decay modes depending on purpose. For the bulk-side decay, the decay constants obtained for various samples showed good agreement 45 with those obtained by the OPP method ${ }^{25-27,31}$, and the effect of the built-in potential on the photoexcited carrier dynamics ${ }^{27}$, as well as the direct detection of photoexcited carrier diffusion ${ }^{28}$, was successfully observed. For the surface-side decay, which enables probing of local dynamics at the surface, even a single${ }_{50}$ atomic-level analysis of the carrier dynamics via in-gap states has been demonstrated ${ }^{25,29}$.

Despite these successes, however, even the conditions for the observation of the two components have not yet been clarified because of the tangled mechanism of the OPP-STM measurement 55 on a semiconductor. In this study, the mechanism of producing OPP-STM spectra from semiconductors was analyzed by comparing model simulation data with experimental data. The optimum conditions required to realize reliable measurement were demonstrated, for the first time, with the validity of the 60 microscopy technique.

First, the fundamental mechanism of OPP-STM is reviewed. In OPP-STM, the sample surface beneath the STM tip is illuminated with a sequence of paired laser pulses with a certain delay time $t_{\mathrm{d}}$ as in the OPP method, and the change in tunneling current $\Delta I$ is ${ }_{65}$ measured as a function of $t_{\mathrm{d}}$. Here, we also call the two pulses pump and probe pulses, whose intensities are adjusted to be the same. The optical pulses give rise to current pulses in the raw tunneling current $I^{*}$, reflecting the excitation and relaxation of the sample. When $t_{\mathrm{d}}$ is sufficiently long compared with the relaxation 70 time of the excited state, the paired optical pulses with the same intensity independently induce two current pulses with the same height in $I^{*}$. In contrast, when $t_{\mathrm{d}}$ is short and the probe pulse illuminates a sample excited by the pump pulse, the second current pulse may have a different height, depending on $t_{\mathrm{d}}$. The 75 signal $\Delta I$ therefore also depends on $t_{\mathrm{d}}$, because the height difference in the second current pulse changes the temporally averaged value of the tunneling current. Accordingly, the relaxation dynamics of the excited state in the target material, for example, the decay of carriers after excitation by a pump pulse, 


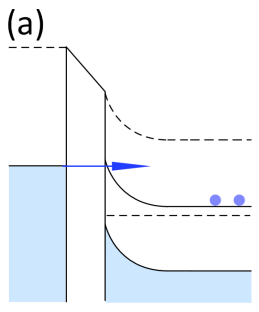

(d)

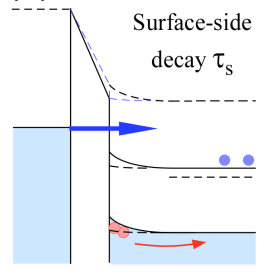

(b)

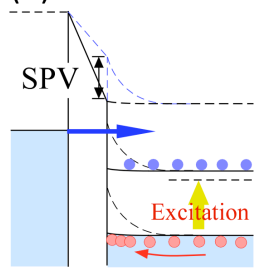

(e)

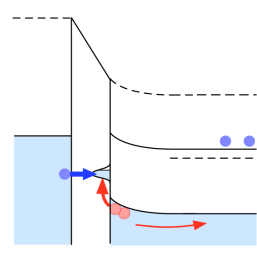

Fig. 1 Schematic illustrations of $1 \mathrm{D}$ band structures in OPP-STM measurement of bare surface ((a) to (d)) and surface with in-gap state (e).

can be probed by STM.

How does the mechanism work in OPP-STM on semiconductors? Figure 1 shows schematic illustrations of the one-dimensional (1D) model of a tunnel junction. Under a reverse 5 bias voltage condition, i.e., a positive bias voltage is applied to an n-type semiconductor, the so-called tip-induced band bending (TIBB) occurs owing to the leakage of the electric field applied between the STM tip and the sample (Fig. 1(a)) ${ }^{33-37}$. When the surface is photoilluminated, the redistribution of photocarriers 10 reduces the electric field and changes the surface potential (surface photovoltage: SPV), and thus increases the effective bias voltage applied to the tunnel junction (Fig. 1(b) $)^{9-11,35-37}$. Consequently, the photoexcitation increases transient tunneling current due to the reduction in the tunneling barrier. The excited

is state subsequently relaxes to the original state via two processes: the decay of photocarriers on the bulk side via recombination, diffusion and drift (bulk-side decay with decay constant $\tau_{\mathrm{b}}$, Fig. 1(c)), and the decay of minority carriers transiently trapped at the surface during photoexcitation (surface-side decay with decay ${ }_{20}$ constant $\tau_{\mathrm{s}}$, Fig. 1(d)). When a probe pulse arrives during the relaxation processes, an additional SPV is induced, which changes the total magnitude of tunneling current depending on $t_{\mathrm{d}}$, providing time-resolved spectra ${ }^{23-29}$. Therefore, bulk-side decay and surface-side decay can be probed by measuring tunneling ${ }_{25}$ current as a function of delay time ${ }^{25}$.

\section{Experimental}

To observe these processes in detail, we measured the laser intensity dependence of OPP-STM spectra. A clean GaAs(110) surface (Si-doped, $1 \times 10^{17} \mathrm{~cm}^{-3}$ ) was prepared by cleaving a GaAs 30 sample in a vacuum $\left(\sim 5 \times 10^{7} \mathrm{~Pa}\right)$. In the OPP-STM system we have developed, pulse trains are generated by two synchronized Ti:sapphire lasers with a $90 \mathrm{MHz}$ repetition rate (11 ns intervals) and a pulse width of $140 \mathrm{fs}$. The relative timing of the two pulse trains, which provides a delay time that ranges from 0 to $11 \mathrm{~ns}$, is

${ }_{35}$ controlled by the synchronizing circuit. Each train is guided to a pulse picker that can selectively transmit one pulse per 90 pulses, resulting in the reduced repetition rate of $1 \mathrm{MHz}$. The pulse selection enables the production of an additional longer delay time that can be adjusted in multiples of $11 \mathrm{~ns}$. Consequently, $t_{\mathrm{d}}$

40 can be adjusted continuously from zero to a large value as needed. Furthermore, the rectangular modulation of $t_{\mathrm{d}}$ allows the measurement of the absolute value of $\Delta I\left(t_{\mathrm{d}}\right)=I\left(t_{\mathrm{d}}\right)-I(\infty)$, where $I(\infty)$ is the tunneling current for a delay time sufficiently long for the excited state to be relaxed ${ }^{25}$. Delay-time modulation as the ${ }_{45}$ reference signal for lock-in detection, instead of laser-intensity modulation, was used to clear thermal effects caused by such as thermal expansion of STM tip and sample ${ }^{20,25}$.

\section{Results and discussion}

Figure 2(a) shows a series of laser-intensity dependent spectra so obtained with a laser repetition rate of $1 \mathrm{MHz}$ and a maximum delay time of $500 \mathrm{~ns}$. The spectra are symmetric with respect to the $t_{\mathrm{d}}=0$ axis, indicating that the intensities of the pump and probe laser pulses were adjusted well to the same value. The laser intensity was changed using filters and normalized with respect to 55 the maximum value of $4 \mathrm{~mW}$.

For high intensities (1 and 0.5), a fast component, which corresponds to the bulk-side decay, was clearly observed near $t_{\mathrm{d}}=$ 0 in addition to the slow component, while only the slow component (surface-side decay) was observed for low intensities ${ }_{60}(\leqq 0.2)$. The spectra were well fitted with a combination of two exponential functions, each with a single decay constant, and the amplitudes and decay constants obtained by fitting are shown in Figs. 2(b) and 2(c), respectively, as a function of the logarithm of laser intensity.

${ }_{65}$ For the fast component, we carried out fitting using the spectra measured with the synchrolock system (not shown here) to obtain accurate values. For the spectra obtained at low laser intensities ( $\leqq 0.05$ in Fig. 2(a)), a slope still remained at $500 \mathrm{~ns}$, indicating that the dynamics were not in a steady state even at $500 \mathrm{~ns}$. To 70 make the fitting more accurate, the measurements were carried out again with a repetition rate of $0.5 \mathrm{MHz}$ and $t_{\mathrm{dmax}}=1000 \mathrm{~ns}$, which is the reason for the two curves showing $\tau_{\mathrm{s}}$ in Fig. 2(b).

The surface-side decay was observed over the entire range of laser intensities with a broad maximum (Fig. 2(b)) and its decay 75 constant $\tau_{\mathrm{s}}$ decreased with increasing laser intensity (Fig. 2(c)), while the bulk-side decay emerged only for high laser intensities and its decay constant $\tau_{\mathrm{b}}$ showed no apparent laser intensity dependence (Figs. 2(b) and 2(c)).

From the model discussed above, a change in laser intensity 80 changes the magnitude of SPV, which, therefore, is considered to play basic roles in both the modes. In fact, to reflect bulk-side decay, a fast response of SPV to carrier dynamics is necessary, and therefore, a high laser intensity that flattens TIBB is required, as experimentally observed. On the other hand, surface-side ${ }_{85}$ decay can be observed over the entire laser intensity range in which SPV is produced, as expected, but its decay constant is considered to change continuously with the relaxation of SPV in a wider range with increasing laser intensity, instead of the exponential decay observed in the spectra ${ }^{9,10}$.

90 To observe the process for producing $\tau_{\mathrm{s}}$ in more detail, we carried out measurements with the control of the SPV baseline by adjusting the extinction rate of the Pockels cell, with the laser intensity fixed at a certain value. Namely, the sample surface was additionally illuminated with optical pulses other than the pump 
(a)

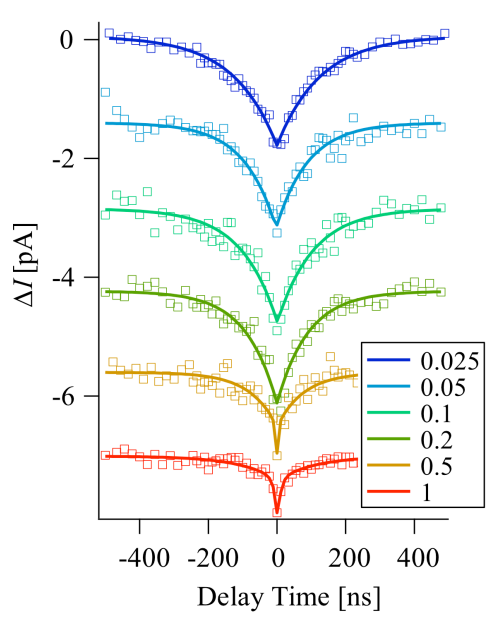

(b)

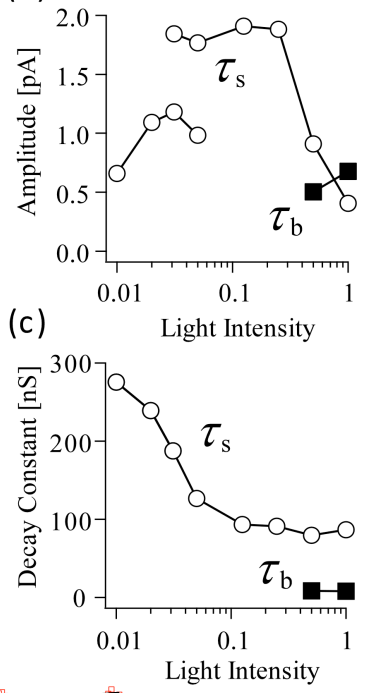

(d)

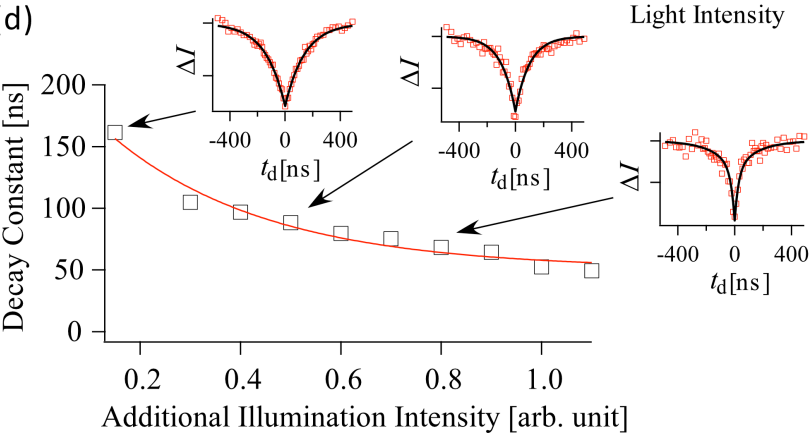

Fig. 2 (a) Laser-intensity dependence of OPP-STM spectra obtained for GaAs(110) sample (set point: $V_{\mathrm{s}}=1 \mathrm{~V}, I_{\mathrm{t}}=100 \mathrm{pA}$ ). The laser intensity was normalized with the maximum value of $4 \mathrm{~mW}$. Amplitudes (b) and decay constants (c) were obtained by the exponential fitting of the spectra in (a) (Typical six spectra are shown in (a)). (d) Decay constant as a function of additional laser intensity.

and probe pulses ( 89 of 90 pulses) with a transmission rate of $R_{\mathrm{T}}$. Figure 2(d) shows the result, where three STM spectra with exponential fitting are shown together. Each OPP-STM spectrum shows exponential decay with a single decay constant, which 5 decreases with increasing $R_{\mathrm{T}}$. How does this work?

To understand the observed results, we carried out simulations on OPP-STM spectra by considering five key factors: (1) absorption bleaching, (2) the logarithmic relationship between carrier density and SPV, (3) the change in tunneling barrier 10 height, (4) the integration process for repeated signals in measurements, and (5) control of the SPV baseline with additional illumination. The effects of these factors were introduced as follows: (1) $N_{\text {eff }}(t)=N_{\text {ex }}\left[1-\left(n_{\mathrm{b}}(t) / n_{0}\right)\right]$, $V_{\mathrm{SPV}}(t)=\left(1 / k_{\mathrm{B}} T\right) \ln \left[1+A n_{\mathrm{s}}(t) / n_{\mathrm{dop}}\right] \quad 9-11$, $I^{*} \propto I_{0} \sqrt{\phi / \phi_{0}}\left(V_{\mathrm{S}}-V_{T}+V_{\mathrm{S}}\right)$ $\left.V_{\mathrm{s}}+V_{\mathrm{TIBB}}-V_{\mathrm{SPV}}\right)$, and (4) $\Delta I\left(t_{\mathrm{d}}\right)=\int I^{*}\left(t_{\mathrm{d}}, t\right) d t-\int I^{*}(\infty, t) d t$, where $N_{\mathrm{ex}}:$ carrier density excited by the first pump pulse, $N_{\text {eff }}(t)$ : carrier density excited by the optical pulses at $t, n_{0}$ : parameter for modulating the effect of laser pulses, $n_{\mathrm{b}}(t)$ : bulk-side carrier 20 density $n_{\text {dop }}$ : dopant density $\left(1 \times 10^{17} \mathrm{~cm}^{-3}\right), I_{0}$ : current, $W$ : work function of the STM tip (4.54 eV for tungsten tip), $\chi$ : electron affinity (4.07 eV for GaAs), $\phi$ : tunnel barrier height, $\phi_{0}$ : (a)

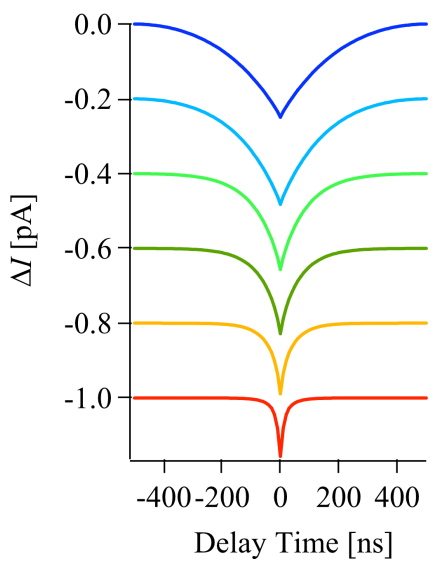

(d)
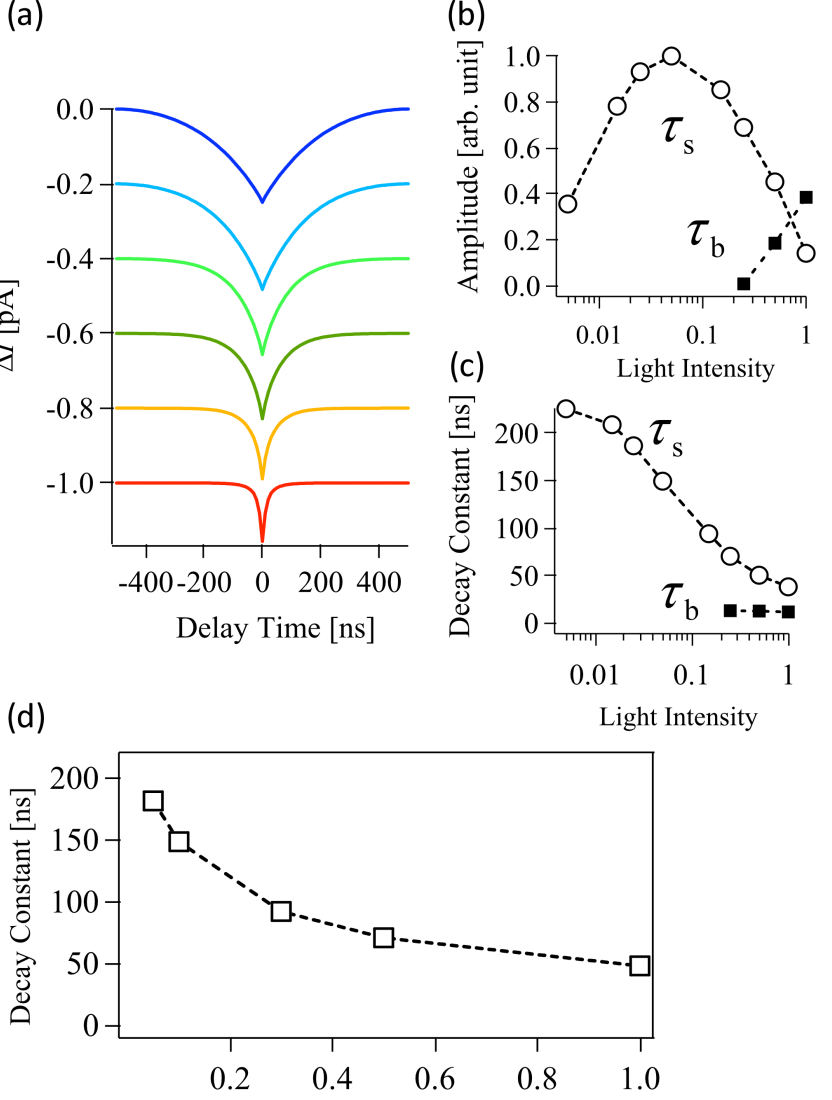

Additional Illumination Intensity [arb. unit]

Fig. 3 Results of simulations carried out for comparison with experimental results shown in Fig. 2. Simulations shown in (a) to (c) were carried out at eight points corresponding to those in Figs. 3(a) to 3(c) (Six of them are shown in (a)). The dependence of the additional illumination intensity of the surface side decay shown in (d) was calculated for five intensities and compared with the experimental results shown in Fig. 2(d). The parameters used were $V_{\mathrm{s}}=1 \mathrm{~V}, I_{0}=50 \mathrm{pA}, N_{\mathrm{ex}}=2 \times 10^{14} \mathrm{~cm}^{-3}, n_{0}=10^{18}$ $\mathrm{cm}^{-3}, \tau_{\mathrm{d}}=1 \mu \mathrm{s}, n_{0}{ }^{\prime}=2 \times 10^{15} \mathrm{~cm}^{-3}, \tau_{\mathrm{b}}=10 \mathrm{~ns}$ and $\mathrm{A}=10^{5}$.

normalizing factor, $V_{\mathrm{s}}$ : bias voltage, $V_{\mathrm{TIBB}}$ : tip-induced band bending, $A$ : constant, $k_{\mathrm{B}}$ : Boltzmann's constant, $T$ : temperature, $25 \mathrm{~m}$ : electron mass, $\hbar$ : Dirac's constant, and z: tip sample distance $(1 \mathrm{~nm})$. (5) The additional illumination was adjusted by changing $R_{\mathrm{T}}$ of the optical pulses arriving at the sample every $10 \mathrm{~ns}$.

We considered two decay modes for $n_{\mathrm{s}}(t)$ : (1) exponential decay for a high laser intensity via recombination in bulk-side decay 30 (decay rate: $1 / \tau_{\mathrm{b}}$ ) and (2) a thermal activation process depending on SPV [decay rate: $1 / \tau_{\mathrm{d}}(t), \tau_{\mathrm{d}}(t)=\tau_{\mathrm{d}} \cdot \exp \left(-V_{\mathrm{SPV}}(t) / k_{\mathrm{B}} T\right)$ ], where $\tau_{\mathrm{d}}$ is the relaxation lifetime under a dark condition with the activation energy of $V_{\text {TIBB }}$ induced by the sample bias voltage $V_{\mathrm{S}}$. The differential equation for $n_{\mathrm{s}}(t)$ is

35

$$
\frac{d n_{\mathrm{s}}(t)}{d t}=-\frac{n_{\mathrm{s}}(t)}{\tau_{\mathrm{d}} \cdot \exp \left(-V_{\mathrm{SPV}}(t) / k_{\mathrm{B}} T\right)} \cdot\left(\frac{n_{0}{ }^{\prime}-n_{\mathrm{b}}(t)}{n_{0}{ }^{\prime}}\right)-\frac{n_{\mathrm{s}}(t)}{\tau_{\mathrm{b}}} \cdot \exp \left(-\frac{t}{\tau_{\mathrm{b}}}\right)
$$

Here, the term $\left[\left(n_{0}{ }^{\prime}-n_{\mathrm{b}}(t)\right) / n_{0}{ }^{\prime}\right]$ delays the activation process (first term) compared with the second term ( $n_{0}$ ' is a constant). The 40 contribution of the second term for a high laser intensity 
decreases with $\exp \left(-t / \tau_{\mathrm{b}}\right)$. For the other parameters, the following values were used: $V_{\mathrm{s}}=1 \mathrm{~V}$ and $V_{\mathrm{TIBB}}=0.3 \mathrm{~V}$ (calculated using Feenstra's formula ${ }^{38}$ for $V_{\mathrm{s}}$ ).

First, to examine the model discussed above, the laser intensity 5 dependences of the two components shown in Fig. 2 were calculated using eq. (1) with the transmission rate $R_{\mathrm{T}}$ of $1 \%$, which was the condition used in our previous experiment and that shown in Fig. 2 because the lowest $R_{\mathrm{T}}$ value is $1 \%$ for our system. Simulations were carried out at eight points corresponding to

10 those in Fig. 2. Figure 3 shows the results obtained, which exhibit excellent agreement with the experimental results shown in Fig. 2, despite the tangled mechanism with various nonlinearities in the measurement steps, supporting the validity of the model. Since a high laser intensity is necessary for measuring the bulk-side 15 decay and there is a limitation of the laser power, the bulk-side decay was observed for only two laser intensities, as shown in Fig. 2. However, according to the simulation, the amplitude ratio of the bulk-side decay for the third laser intensity to that for the second one is $\sim 1 / 25$, as shown in Fig. 3(b). The fact that the bulk20 side decay for the third laser intensity could not be observed experimentally, as shown in Figs. 2(b) and 2(c), is consistent with the simulation results. For laser intensities higher than those in Figs. 2 and 3, the measurement was beyond the limitation of our laser system. More complex and interesting phenomena are ${ }_{25}$ expected for the carrier dynamics induced by higher-intensity laser excitation, which will be an interesting and important research target of this microscopy technique in the future.

Next, to observe the effect of each step in more detail, the relationship among the four steps in the measurement involving ${ }_{30} n_{\mathrm{s}}(t), V_{\mathrm{SPV}}(t), I^{*}(t)$, and $\Delta I(t)$ was examined. Figures $4(\mathrm{a})$ to $4(\mathrm{~d})$ show the results obtained for five values of $R_{\mathrm{T}}(0 \%, 0.1 \%$. $0.5 \%$,

(a) $n_{\mathrm{s}}$

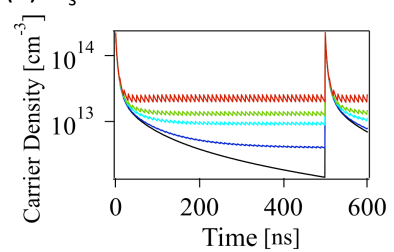

(b) $V_{\mathrm{SPV}}$

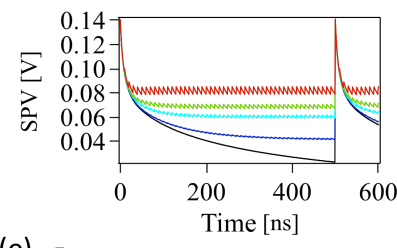

(e) $\tau_{\mathrm{b}}$

(c) $I^{*}$

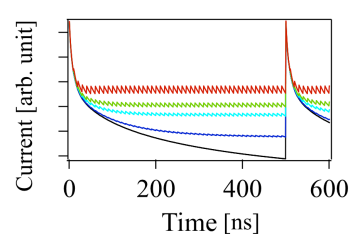

(d) $\Delta l$
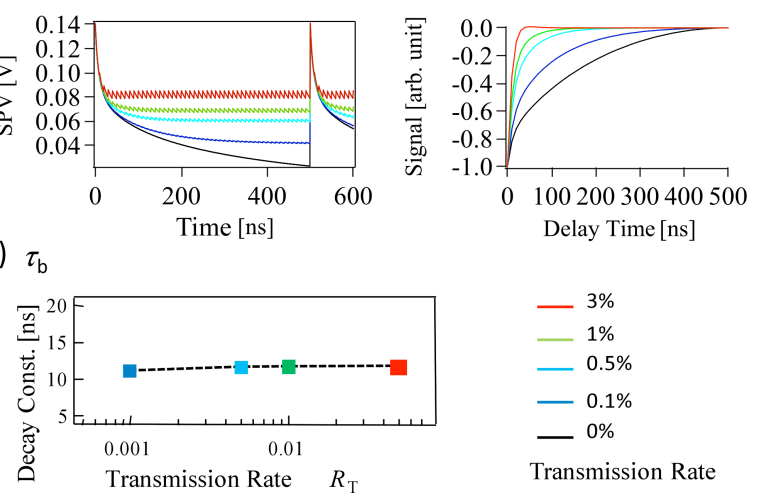

$$
\begin{aligned}
& -{ }^{3} \% \\
& -1 \% \\
& -0.5 \% \\
& -0.1 \% \\
& -0 \% \\
& \text { Transmission Rate }
\end{aligned}
$$

Fig. 4 Simulation results of five transmission rates $R_{\mathrm{T}}$ for four processes in OPP-STM measurement: (a) $n_{\mathrm{s}}(\mathrm{t})$, (b) $\operatorname{SPV}(t)$, (c) $I^{*}(t)$, and (d) $\Delta I(t)$ (e) Transmission rate dependence of $\tau_{\mathrm{b}}$. The parameters used were $V_{\mathrm{s}}=1$ $\mathrm{V}, I_{0}=50 \mathrm{pA}, N_{\mathrm{ex}}=2 \times 10^{14} \mathrm{~cm}^{-3}, n_{0}=10^{18} \mathrm{~cm}^{-3}, \tau_{\mathrm{d}}=1 \mu \mathrm{s}, n_{0}{ }^{\prime}=2 \times 10^{15}$ $\mathrm{cm}^{-3}, \tau_{\mathrm{b}}=10 \mathrm{~ns}$ and $\mathrm{A}=10^{5}$.
$1.0 \%$ and $3 \%$ ). Simulations were carried out for these $R_{\mathrm{T}}$ values to determine under which condition the measurement can be optimized. Although the spectra obtained for $R_{\mathrm{T}}=0$ could not be 35 fitted by exponential functions with a constant decay rate, it became possible for a finite $R_{\mathrm{T}}$. This is due to the fact that the effect of the long-range relaxation of $n_{\mathrm{s}}(t)$ with the timedependent decay constant $\tau_{\mathrm{s}}(t)$ is reduced. When the sample surface is additionally illuminated every $10 \mathrm{~ns}$ by optical pulses ${ }_{4}$ with transmission rate $R_{\mathrm{T}}$, the SPV baseline increases and the magnitude of the change in SPV decreases, depending on $R_{\mathrm{T}}$. This, in conjunction with the nonlinearities discussed above, changes the decays of SPV and $I^{*}$ into exponential decay. Thereby, despite the fact that the change in $n_{\mathrm{s}}(t)$ includes a 45 thermal excitation process, the STM signal $\Delta I(t)$ is expressed by a combination of two exponential functions. The $R_{\mathrm{T}}$ dependence of $\tau_{\mathrm{s}}$ shown in Fig. 3(d) expresses the change in the thermal activation process approximated by a constant decay within the narrowed decay range in SPV. By comparing the values of $\tau_{\mathrm{d}}\left(R_{\mathrm{T}}\right)$ ${ }_{50}$ in Figs. 2(d) and $3(\mathrm{~d}), \tau_{\mathrm{d}}$ can be evaluated using the relation $\tau_{\mathrm{d}}(t)=\tau_{\mathrm{d}} \cdot \exp \left(-V_{\mathrm{SPV}}(t) / k_{\mathrm{B}} T\right)$.

Figure 4(e) shows the decay constant of the bulk-side decay, $\tau_{\mathrm{b}}$, as a function of $R_{\mathrm{T}}$. The decay constant slightly increases with increasing $R_{\mathrm{T}}$, and its value ( $\left.\sim 11 \mathrm{~ns}\right)$ is close to that used for the ${ }_{55}$ simulations (10 ns). According to the simulation results, the bulkside decay can be probed efficiently by OPP-STM with the additional light of $R_{\mathrm{T}}=1 \%$. In fact, the decay constants obtained for various samples are in good agreement with those obtained by the OPP method ${ }^{25,27}$. Under high-intensity excitation, various $6_{0}$ interesting and complex phenomena are included in the carrier dynamics, which may be considered when more detailed theoretical analyses of OPP-STM results are carried out.

Lastly, let us analyze the case of OPP-STM analysis on an ingap state, which has been shown in recent papers ${ }^{25,29}$. In the ${ }_{65}$ presence of the in-gap state shown in Fig. 1(e), holes trapped at the surface recombine with the electrons tunneling from the STM tip. When sufficient electrons are provided, the hole capture rate, which becomes the limiting factor of the process, can be obtained

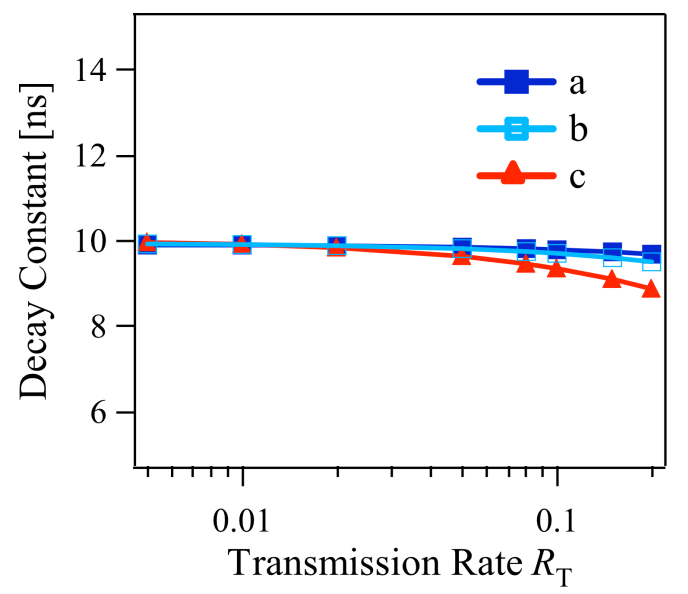

Fig. 5 Simulation results for analysis of hole dynamics via in-gap state shown in Fig. 1(e): decay constant $\tau_{\mathrm{g}}$ as a functions of $R_{\mathrm{T}}$ for three different laser intensities ((a) $5 \times 10^{12}$, (b) $1 \times 10^{13}$, (c) $\left.3 \times 10^{13} \mathrm{~cm}^{-3}\right)$. The parameters used were $V_{\mathrm{s}}=1 \mathrm{~V}, I_{0}=50 \mathrm{pA}, N_{\mathrm{ex}}=2 \times 10^{14} \mathrm{~cm}^{-3}, n_{0}=10^{18}$ $\mathrm{cm}^{-3}, \tau_{\mathrm{d}}=1 \mu \mathrm{s}$ and $\tau_{\mathrm{g}}=10 \mathrm{~ns}$. 
even for a single atomic level ${ }^{29}$. To satisfy the conditions, two methods were used in previous experiments: (1) the tunneling current was increased while the STM tip was moved toward the sample and (2) the laser intensity was decreased ${ }^{25,29}$. Here, the 5 laser intensity was adjusted.

The equation used for simulation is

$$
\frac{d n_{\mathrm{s}}(t)}{d t}=-\frac{n_{\mathrm{s}}(t)}{\tau_{\mathrm{d}} \cdot \exp \left(-V_{\mathrm{SPV}}(t) / k_{\mathrm{B}} T\right)}-\frac{n_{\mathrm{s}}(t)}{\tau_{\mathrm{g}}}
$$

${ }_{10}$ Equation (2) was obtained from eq. (1), in accordance with the measurement condition described above, by assuming $n_{\mathrm{b}}(t) / n_{0}{ }^{\prime}=$ 0 , which produces the first term in eq. (2), and replacing the second term in eq. (1) with $n_{\mathrm{s}}(t) / \tau_{\mathrm{g}}$ because the laser intensity is low and the effect of the in-gap state is dominant. Here, $\tau_{\mathrm{g}}$ is the 15 decay constant of $n_{\mathrm{S}}$ obtained by the recombination of holes with electrons tunneling from the STM tip via the in-gap state, which is assumed as a constant to satisfy the condition required for the tunneling current to be sufficient for the hole capture rate to be the limiting factor of the process.

20 Simulations similar to those in Fig 4 were carried out and the results obtained for the $R_{\mathrm{T}}$ dependence of $\tau_{\mathrm{g}}$ are shown in Fig. 5. Similarly to the case in Fig. 4, fitting by exponential functions with a constant decay rate became possible for a finite $R_{\mathrm{T}}$. The decay constant $\tau_{\mathrm{g}}$ slightly decreases with increasing $R_{\mathrm{T}}$, but its ${ }_{25}$ value corresponds well to the $\tau_{\mathrm{g}}$ used in the simulation $(10 \mathrm{~ns})$. With the transmission rate $R_{\mathrm{T}}$ of $1 \%$, which was confirmed to be a suitable condition for probing bulk-side decay, $\tau_{\mathrm{g}}$ is almost constant $(\sim 10 \mathrm{~ns})$ for the three laser intensities and sufficient for performing a reliable experiment on the analysis of in-gap states. 30 In conjunction with light-modulated scanning tunneling
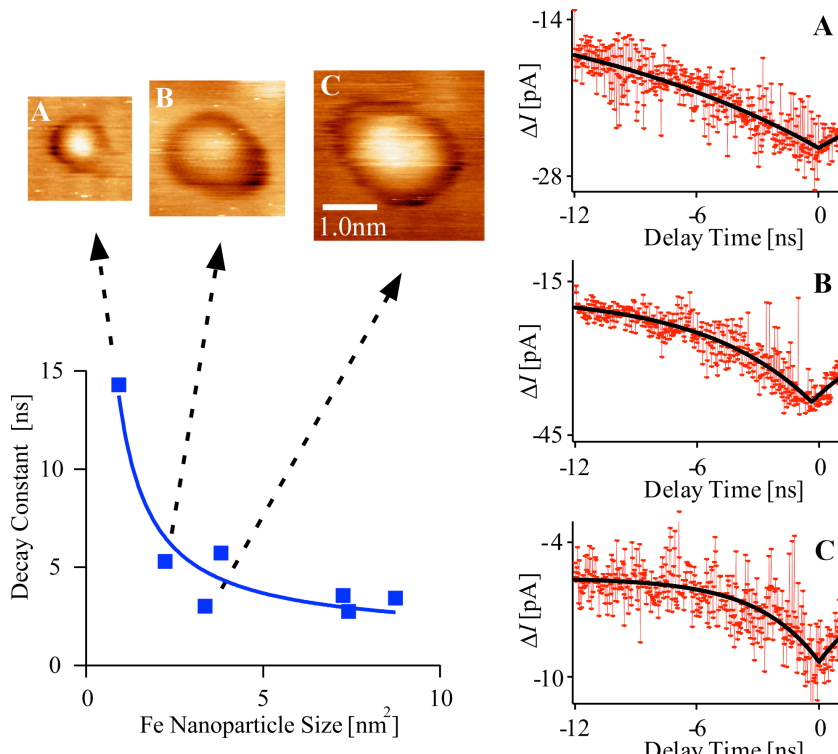

A

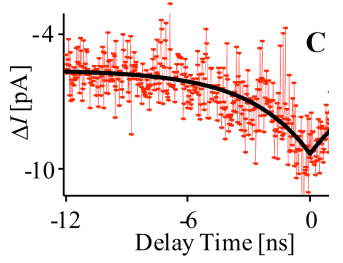

Fig. 6 Decay constant of density of photoinduced hole transiently trapped at the surface as a function of the Fe nanoparticle size on a $\mathrm{GaAs}(110)$ surface. Three spectra with a single exponential fitting curve, which were obtained above the Fe nanoparticles shown by STM images, are shown together (the size of the Fe nanoparticle in image A corresponds to a single Fe atom). spectroscopy $^{36,37}$, single-atomic-level analysis is possible ${ }^{29}$.

Figure 6 shows the experimentally obtained time-resolved OPPSTM signals for Fe nanoparticle/GaAs(110) samples. The in-gap states formed by the structure enhance the recombination of holes 35 transiently trapped at the surface with electrons tunneling from the STM tip at the in-gap states. Exponential spectra were obtained regardless of the Fe nanoparticle size as predicted, three examples of which are shown in Fig. 6, despite the complex processes including various nonlinearities as described above. As 40 the Fe base area increases, the hole capture probability is expected to increase and thus the decay constant decreases, which corresponds well to the obtained results.

Since hole capture rate, for example, is considered to depend on temperature, the activation process, which is included in $\tau_{\mathrm{g}}$, is 45 expected to be analyzed at the atomic level if the temperature dependence of carrier dynamics is measured ${ }^{32}$. Bulk-side decay may also show temperature dependence depending on the type of the sample used. These are interesting research targets for the application of our microscopy technique.

\section{${ }_{50}$ Conclusions}

The probing mechanism of OPP-STM spectra from semiconductors was examined. The conditions required to obtain comprehensive results were demonstrated for the first time. When such conditions are satisfied, transient carrier dynamics can be ${ }_{55}$ probed directly by measuring the tunneling current as a function of delay time, in which decay constants of carrier dynamics can be obtained by the exponential fitting of the spectra without complex calibrations. In this study, we considered a $\operatorname{GaAs}(110)$ sample because a clean surface without in-gap states can be ${ }_{60}$ prepared by cleavage, which can provide a typical example applicable for further experiments ${ }^{39,40}$. Different materials have different characteristics and more complex carrier dynamics may be induced, for example, by a higher-laser-intensity excitation. Further progress in probing more complex phenomena and a ${ }_{65}$ comprehensive understanding may be realized with more detailed theoretical analyses of OPP-STM results obtained for various materials, which is expected to make OPP-STM more applicable and play more important roles in further advances in nanoscale science and technologies.

\section{Acknowledgement}

Support from Japan Society for the Promotion of Science (Grants-in-Aid for Scientific Research) is acknowledged.

\section{Notes and references}

$75{ }^{a}$ University of Tsukuba, Tskuba 305-8571, Japan. Fax:+81-29-853-5276; Tel:+81-29-853-5276; E-mail: hidemi@ims.tsukuba.ac.jp http://dora.bk.tsukuba.ac.jp

${ }^{b}$ On leave from the University of Tokyo

${ }_{80} 1$ G. Binning, H. Rohrer, H, Ch. Gerber and E. Weibel, Phys. Rev. Lett.,1982, 49, 57.

2 R. Wisendanger: Scanning Probe Microscopy and Spectroscopy (Cambridge University Press, Cambridge, 1994).

3 H. J. Mamin, H. Birk, P. Wimmer and D. Rugar, J. Appl. Phys., 1994, $85 \quad 75,161$. 
4 U. Kemiktarak, T. Ndukum, K.C. Schwab and K. L. Ekinci, Nature, $2007,85,450$.

5 G. Nunes, Jr. and M. R. Freeman, Science, 1993, 262, 1029.

6 G. M. Steeves, A. Y. Elezzabi and M. R. Freeman, Appl. Phys. Lett., 1998, 72, 504.

7 D. Botkin, J. Glass, D. S. Chemla, D. F. Ogletree, M. Salmeron and S Weiss, Appl. Phys. Lett., 1998, 72, 504.

8 I. Moult, M. Herve and Y. Pennec, Appl. Phys. Lett., 2011, 98, 233103.

109 R. J. Hamers and D. G. Cahill, Appl. Phys. Lett., 1990, 57, 2031.

10 R. J. Hamers and D. G. Cahill, J. Vac. Sci. Technol., 1991, 9, 514..

11 S. Grafstroöm, J. Appl. Phys., 2002, 91, 1717.

12 S. Loth, M. Etzkorn, C. P. Lutz, D. M. Eigler and A. J. Heinrich, Science, 2010, 329, 1628.

1513 C. Saunus, J. R. Bindel, M. Pratzer and M. Morgenstern, Appl. Phys. Lett., 2013, 102, 051601.

14 U. Kemiktarak, T. Ndukum, K.C. Schwab and K. L. Ekinci: Nature, 2007, 450, 85.

15 N. N. Khusnatdinov, T. J. Nagle and G. Nunes, Appl. Phys. Lett., 1996, 69, 2294.

16 R. H. M. Groeneveld and H. van Kempen, Appl. Phys. Lett., 2000, 77, 4434.

17 R. Giridharagopal, G. E. Rayermann, G. Shao, D. T. Moore, O. G. Reid, A. F. Tillack, D. J. Masiello and D. S. Ginger, Nano Lett., 2012, 12, 893.

18 S. Weiss, D. F. Ogletree, D. Botkin, M. Salmeron and D. S. Chemla: Appl. Phys. Lett., 1993, 63, 2567.

19 O. Takeuchi, R. Morita, M. Yamashita and H. Shigekawa, Jpn. J. Appl. Phys., 2002, 41, 4994.

3020 O. Takeuchi, M. Aoyama, R. Oshima, Y. Okada, H. Oigawa, N. Sano, H. Shigekawa, R. Morita and M. Yamashita, Appl. Phys. Lett., 2004, $\mathbf{8 5}, 3268$.

21 O. Takeuchi, M. Aoyama and H. Shigekawa, Jpn. J. Appl. Phys., $2005,44,5354$.

3522 O. Takeuchi, M. Aoyama, M. Kondo, A. Taninaka, Y. Terada and H. Shigekawa, Jpn. J. Appl. Phys., 2006, 45, 1926.

23 Y. Terada, S. Yoshida, O. Takeuchi and H. Shigekawa, J. Phys. Condens. Matter, 2010, 22, 264008.

24 Y. Terada, M. Aoyama, H. Kondo, A. Taninaka, O. Takeuchi and H. Shigekawa, Nanotechnology, 2007, 18, 044028.

25 Y. Terada, S. Yoshida, O. Takeuchi and H. Shigekawa, Nat. Photonics, 2010, 4, 869.

26 Y. Terada, S. Yoshida, O. Takeuchi and H. Shigekawa, Adv. Opt Tech., 2011, 2011, 510186.

4527 S. Yoshida, Y. Terada, R. Oshima, O. Takeuchi and H. Shigekawa, Nanoscale, 2012, 4, 757.

28 S. Yoshida, M. Yokota, O. Takeuchi, Y. Mera, and H. Shigekawa, Appl. Phys. Exp., 2013, 6, 016601.

29 S. Yoshida, M. Yokota, O. Takeuchi, H. Oigawa, Y. Mera and H. Shigekawa, Appl. Phys. Exp., 2013, 6, 032401.

30 S. W. Wu and W. Ho, Phys. Rev. B., 2010, 82, 085444.

31 J. Shah, Ultrafast Spectroscopy of Semiconductors and Semiconductor Nanostructures (Berlin: Springer 1999).

32 A. Richardella, D. Kitchen, and A. Yazdani: Phys. Rev. B., 2009, 80, 045318.

33 R. M. Feenstra, Y. Dong, M. P. Semtsiv and W. T. Masselink, Nanotechnology, 2007, 18, 044015.

34 R. M. Feenstra, Surf. Sci. 2009, 603, 2841.

35 M. McEllistrem, G. Haase, D. Chen and R. J. Hamers, Phys.Rev. Lett.,1993, 70, 2471.

36 S. Yoshida, Y. Kanitani, R. Oshima, Y. Okada, O. Takeuchi and H. Shigekawa, Phys. Rev. Lett., 2007, 98026802.

37 O. Takeuchi, S. Yoshida and H. Shigekawa, Appl. Phys. Lett., 2004, 84, 3645 .

6538 R. M. Feenstra, J. Vac. Sci. Technol. B, 2003, 21, 2080.

39 R. M. Feenstra, J. A. Stroscio, J. Tersoff and A. P. Fein, Phys. Rev. Lett. 1987, 58, 1192.

40 R. M. Feenstra and J. A. Stroscio, J. Vac. Sci. Technol. B., 1987, 5, 923. 

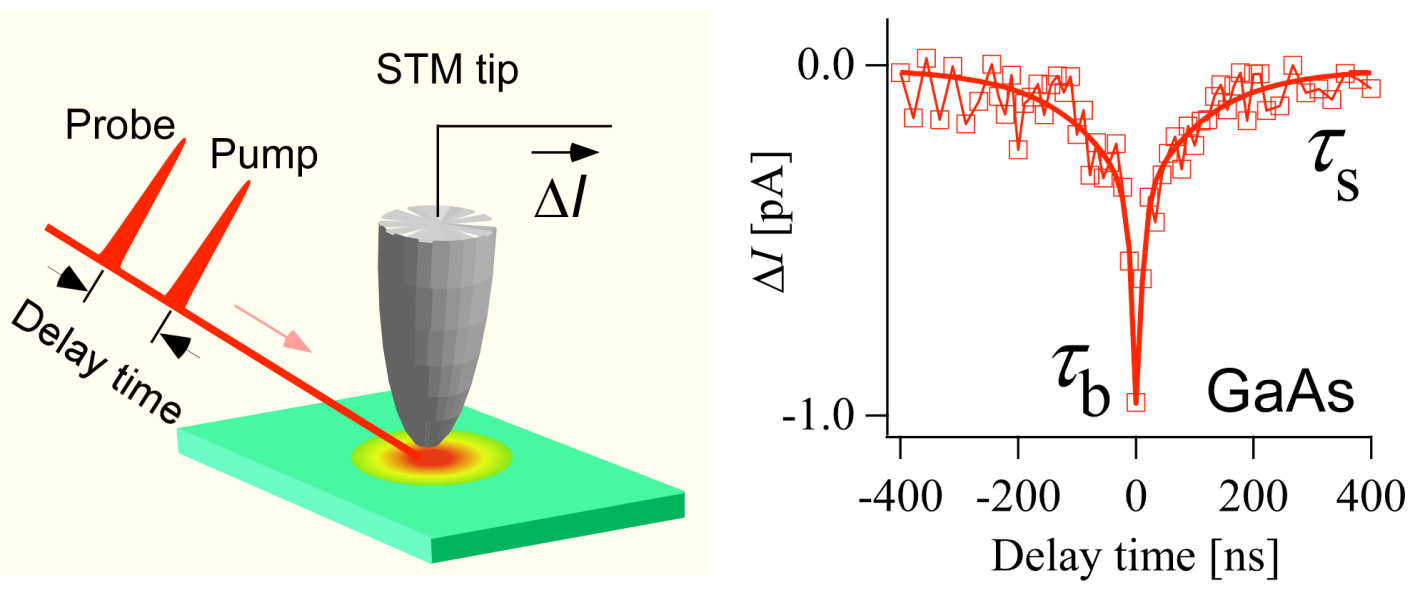

We first demonstrate a comprehensive understanding of the tangled mechanism that produces optical pump-probe scanning tunneling microscopy spectra from semiconductors. 